\title{
IMPLICACIONES DEL ÓXIDO NÍTRICO Y OTRAS MOLÉCULAS CON POTENCIAL REDOX EN EL GLAUCOMA PRIMARIO DE ÁNGULO ABIERTO
}

\section{INVOLVEMENT OF NITRIC OXIDE AND OTHER MOLECULES WITH REDOX POTENTIAL IN PRIMARY OPEN ANGLE GLAUCOMA}

\author{
ZANÓN-MORENO V¹, PONS $\mathrm{S}^{2}$, GALLEGO-PINAZO R ${ }^{3}$, GARCÍA-MEDINA J ${ }^{4}$, VINUESA I ${ }^{5}$, \\ VILA BOU V ${ }^{6}$, PINAZO-DURÁN MD 6
}

\section{RESUMEN}

Objetivos: El óxido nítrico (ON) y otras moléculas con potencial red-ox están relacionados con mecanismos de transmisión de señales, incluyendo la regulación de la permeabilidad vascular y la homeostasis. Nos proponemos investigar la presencia de ON y de especies reactivas del oxígeno (EROx) en el humor acuoso y su relación con los mecanismos patogénicos del glaucoma primario de ángulo abierto (GPAA).

Métodos: Se ha analizado bioquímicamente el humor acuoso, que fue extraído al comienzo de la cirugía de segmento anterior, de dos grupos de individuos: 1) pacientes programados para trabeculectomía de Watson, por empeoramiento de los parámetros de riesgo del glaucoma (GG; $n=60$ ) y 2 ) grupo comparativo de sujetos intervenidos mediante faco-
ABSTRACT

Purpose: Nitric oxide (NO) and other molecules with redox potential are involved in cell signalling, including endothelial-dependent relaxation and the maintenance of vascular homeostasis. We investigated the availability of NO and the formation of reactive oxygen species (ROS) in the aqueous humour and its relationship to the pathogenic mechanisms of primary open-angle glaucoma (POAG).

Methods: We analysed biochemically aqueous humour samples from patients having anterior segment surgery that were divided into two separate groups: 1) patients having a Watson's trabeculectomy because of worsening of the glaucoma evolution (GG; $n=60)$, and 2) a comparative group of individuals having phacoemulsification for noncomplicated cataracts $(\mathrm{CG} ; \mathrm{n}=60)$. Enzymatic-colo-

Recibido: 18/12/06. Aceptado: 14/5/08.

Unidad de Investigación Oftalmológica Santiago Grisolía. Hospital Universitario Dr. Peset. Valencia. España.

1 Doctor en Medicina. Universidad de Valencia. Unidad de Investigación Oftalmológica Santiago Grisolía. Hospital Universitario Dr. Peset. Valencia.

${ }^{2}$ Licenciado en Biología. Unidad de Investigación Oftalmológica Santiago Grisolía. Hospital Universitario Dr. Peset. Valencia.

3 Licenciado en Medicina. Unidad de Investigación Oftalmológica Santiago Grisolía. Hospital Universitario Dr. Peset. Valencia. Hospital universitario La Fe. Valencia.

4 Doctor en Medicina. Unidad de Investigación Oftalmológica Santiago Grisolía. Hospital Universitario Dr. Peset. Valencia. Hospital La Inmaculada Huercal Overa. Almería.

5 Doctor en Medicina. Servicio de Oftalmología del Hospital Punta de Europa. Algeciras. Cádiz.

6 Doctor en Medicina. Unidad de Investigación Biomédica Departamento Sanitario 10. Valencia. Unidad de Investigación Oftalmológica Santiago Grisolía. Hospital Universitario Dr. Peset. Valencia.

Este trabajo ha sido financiado parcialmente por el Instituto de Salud Carlos III, Proyecto FIS PI061962, por laboratorios MSD-Chibret y por laboratorios THEA. Parte de los resultados han sido presentados a los Congresos anuales de la ARVO (Fort Lauderdale, Florida, USA; 2007), ISPOR (Dublín, Irlanda; 2007) y de la AAO (Nueva Orleans, Louisiana, 2007).

Correspondencia:

María Dolores Pinazo-Durán

Unidad de Investigación Biomédica

Avenida Gaspar Aguilar, 90

46017 Valencia

España

E-mail: pinazo_mar@gva.es 
emulsificación para extracción de las cataratas no complicadas (GC; $\mathrm{n}=60)$. Las determinaciones del ON, especies reactivas del oxígeno (EROx) y la actividad antioxidante total (AOXT) fueron llevadas a cabo mediante técnicas enzimático-colorimétricas.

Resultados: Se ha observado un aumento significativo de la concentración de ON y de EROx (MDA) $\mathrm{y}$, paralelamente, una disminución significativa de antioxidantes (AOXT) en el humor acuoso de los pacientes glaucomatosos en comparación con los individuos del GC.

Conclusiones: Las anomalías en la disponibilidad de ON y la concomitancia de estrés oxidativo en el humor acuoso sugieren que estas biomoléculas pueden utilizarse como marcadores de evolución de la enfermedad glaucomatosa y ayudar a establecer individualizadamente el riesgo de pérdida de visión.

Palabras clave: Glaucoma, óxido nítrico, especies reactivas del oxigeno, antioxidantes, estrés oxidativo. rimetric methods were used to analyse the NO concentration, the lipid peroxidation by-product malondialdehyde (MDA) and the total antioxidant status (TAS). Statistical analysis was carried out in the two groups by means of the SPSS 15.0 programme.

Results: A significant increase in both the NO concentration $(\mathrm{p}<0.05)$ and MDA levels $(\mathrm{p}<0.05)$, and a significant decrease of the TAS $(\mathrm{p}<0.05)$ were detected in the GG versus the CG samples.

Conclusions: Changes in NO availability and the concomitance of oxidative stress in the aqueous humour of the POAG patients may be useful markers for identifying those at risk of glaucoma progression and visual loss (Arch Soc Esp Oftalmol 2008; 83: 365-372).

Key words: Glaucoma, nitric oxide, reactive oxygen species, antioxidants, oxidative stress.

\section{INTRODUCCIÓN}

El humor acuoso se genera y evacua de forma constante y equilibrada en las estructuras del segmento anterior del ojo para su propia nutrición y el mantenimiento del tono ocular (1). La regulación de la presión intraocular (PIO) depende del equilibrio de complejos mecanismos implicados en la producción y drenaje del humor acuoso, entre ellos la estabilidad y supervivencia de los fenotipos celulares implicados y el mantenimiento de la homeostasis. El diagnóstico precoz de la hipertensión ocular (HTO) es esencial para prevenir el glaucoma. El glaucoma es aún la principal causa de ceguera irreversible a nivel mundial, con una incidencia del $2,4 \%$. Se estima que 66,8 millones de personas padecen glaucoma, de los cuales 6,7 millones presentan ceguera bilateral (2). En España existen unas 300.000 personas con glaucoma diagnosticado y aproximadamente 700.000 personas lo padecen sin diagnosticar. Aunque se han identificado factores de riesgo para la enfermedad glaucomatosa $(3,4)$ y se ha avanzado en el tratamiento clínico-quirúrgico de ésta, aún no se ha podido evitar la progresión incoercible del glaucoma y la ceguera por atrofia óptica.

El óxido nítrico $(\mathrm{ON})$ es un gas hidrofóbico de vida corta, producido endógenamente y que posee una estructura química relativamente sencilla. El ON se sintetiza de forma endógena a partir de la L- arginina, el oxígeno y la NADPH, según aparece en las reacciones siguientes mediadas por las enzimas óxido nítrico sintasas (ONSs), y de las cuales se han identificando tres isoformas: NOS1 (también llamada NOS neuronal, nNOS o NOS I), NOS2 (NOS inducible, iNOS ó NOS II) y NOS3 (NOS endotelial, eNOS o NOS III). Todos los subtipos producen ON, L-citrulina y NADP+ precisando también el grupo hemo y el $\mathrm{Ca}^{2+} /$ calmodulina como cofactores $(5,6)$.

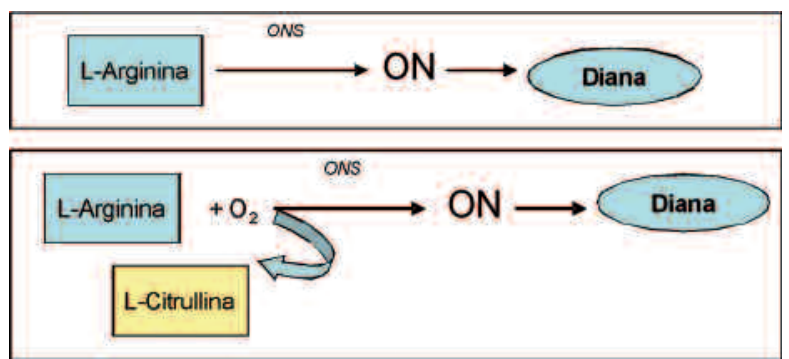

A finales de los años 70 se demostró que los efectos vasodilatadores de la nitroglicerina y otros nitratos estaban mediados por el ON y que éste último podía activar la enzima guanilil ciclasa soluble en las células musculares lisas de los vasos sanguíneos. Esto permitió conocer los mecanismos de síntesis de GMP cíclico por parte del enzima específico. Casi al mismo tiempo, se conoció que la relajación de los vasos sanguíneos dependiente de la acción de la acetilcolina necesitaba un endotelio vascular intacto y así se pensó que debía existir una sustan- 
cia específica, que cuando era liberada por las células endoteliales pudiera actuar sobre las células musculares lisas, es decir lo que consideraríamos un «factor de relajación derivado de endotelio vascular» (7). En los años siguientes se llegó a la conclusión de que esta molécula era el ON, alcanzando la evidencia de que las células endoteliales producían el $\mathrm{ON}$ en cantidad suficiente para explicar la relajación observada. Estos hallazgos confirmaron las hipótesis previas, concediéndose en 1998 el Premio Nobel de Fisiología y Medicina a Robert Furchgott, Louis Ignarro y Ferid Murad, por el descubrimiento de la función del $\mathrm{ON}$ como molécula señalizadora en el sistema cardiovascular y su función para el mantenimiento de la salud (8-10). El ON se considera un mensajero fundamental para los sistemas de transducción de señales en el cerebro y aparato cardiovascular. Aunque se identificaron los enzimas sintetizadores en el cerebro (NOS1), y posteriormente en macrófagos (NOS2) y en el endotelio (NOS3), estos enzimas se han encontrado también en otras células del organismo, participando en diversos procesos fisiológicos y patológicos, incluyendo las enfermedades oculares, entre las que se ha descrito su implicación en las cataratas, uveítis y glaucoma (11-13).

El estrés oxidativo es resultado de uno de los siguientes procesos: 1) aumento en la formación y actividad de oxidantes, 2) reducción de los mecanismos de defensa antioxidante, o 3) fracaso de los mecanismos reparadores del daño oxidativo (14). Las especies reactivas del oxígeno (EROx) están constituidas por los radicales libres, aniones reactivos e inestables que contienen átomos de oxígeno, o moléculas que contienen átomos de oxígeno que puede producir radicales libres o que son químicamente activadas por éstos. Entre las EROx son las más relevantes el radical hidroxilo, anión superóxido, peróxido de hidrógeno y el peroxinitrito. La mayor producción de ROS acontece durante la respiración aerobia, aunque también son formadas en los peroxisomas (oxidación de ácidos grasos) y microsomas (sistema citocromo P450 y metabolismo de xenobióticos), la fagocitosis de agentes patógenos o de lipopolisacáridos, el metabolismo de la arginina, y algunos tejidos específicos (15). En condiciones normales, las EROx se eliminan principalmente por la acción de los enzimas superóxido dismutasa (SOD), catalasa y glutation peroxidasa (GSHPx). La formación y acumulación de EROx daña las células y tejidos mediante el ataque a macromoléculas, como los ácidos grasos poliinsaturados de los lípidos de las membranas, las proteínas esenciales, y los ácidos nucleicos16. Diversas enfermedades han sido relacionadas etiopatogénicamente con las EROx, y además el estrés oxidativo forma parte de los mecanismos lesivos de la enfermedad de Alzheimer, la enfermedad de Parkinson, el cáncer y el envejecimiento. En cuanto al sistema visual, también se han implicado las EROx en la etiopatogenia de diversos procesos oftalmológicos, principalmente las cataratas, degeneración macular asociada a la edad y vítreo-retinopatías (17-19).

Aplicando todos estos conceptos a nuestra línea de investigación en glaucoma primario de ángulo abierto (GPAA), e intentando identificar moléculas implicadas en la progresión de la enfermedad glaucomatosa, y estudiando su posible utilización como marcadores del curso del glaucoma, hemos diseñado el presente estudio con el fin de determinar, mediante métodos enzimático-colorimétricos, la presencia del $\mathrm{ON}$ y las actividades oxidativa y antioxidante en el humor acuoso de pacientes con GPAA sometidos a cirugía antiglaucomatosa.

\section{SUJETOS, MATERIAL Y MÉTODOS}

Se han seleccionado 120 pacientes de ambos sexos que acudieron a los controles indicados por los oftalmólogos de la Sección de Glaucoma en las consultas de oftalmología de los hospitales de referencia y que se incluyeron consecutivamente. Tras ser evaluados convenientemente mediante los nomogramas para cálculo de riesgo de progresión del GPAA, según datos de Vinuesa-Silva y cols. todavía no publicados (en revisión Arch Soc Esp Oftalmol), basados en factores de riesgo individual, las pruebas funcionales y los hallazgos morfológicos, se consideraron pacientes con riesgo de pérdida de visión por causa de su enfermedad y por ello fueron destinados a cirugía antiglaucomatosa, mediante la técnica de trabeculectomía de Watson $(n=60)$. Un grupo comparativo de sujetos con cataratas $(\mathrm{GC} ; \mathrm{n}=60)$, programados para extracción de su catarata mediante la técnica de facoemulsificación e implante de lente intraocular, fueron incluidos en este estudio y analizados en paralelo al GG. Los criterios de inclusión y exclusión de los sujetos del estudio se especifican en la tabla I.

En el primer paso de la intervención en ambos grupos de pacientes y a través de la paracentesis de 
Tabla I. Criterios de inclusión y exclusión de los sujetos del estudio

GRUPO GLAUCOMA

INCLUSIÓN

EXCLUSIÓN

40 - 90 años. Ambos Sexos

Glaucoma Primario de Ángulo Abierto

Consentimiento y Colaboración

Hipertensión arterial

INCLUSIÓN

40 - 90 años. Ambos Sexos

Cataratas

Consentimiento y Colaboración
Edad inferior a 40 o superior a 90 años

Glaucoma diferente de PAA

Otra patología ocular

GRUPO CATARATAS

Edad inferior a 40 o superior a 90 años

Otra patología ocular

Hipertensión arterial servicio se introdujo en la cámara anterior una cánula de Rycroff unida a jeringa de insulina, absorbiendo un volumen aproximado de 0,1-0,2 $\mu \mathrm{L}$ de humor acuoso, evitando colapsar la cámara anterior. Estas muestras se depositaron directamente en criotubos estériles que fueron etiquetados y depositados en congelador $\mathrm{a}-20^{\circ} \mathrm{C}$ durante 1 hora. Posteriormente se transportaron y almacenaron hasta su procesamiento en cajas clasificadoras en un ultracongelador $\left(-85^{\circ} \mathrm{C}\right)$.

Las técnicas bioquímicas que se han realizado en todas las muestras de humor acuoso han sido las siguientes:

1) Determinación de la concentración total de óxido nítrico: Mediante un preparado comercial de $R \& D$ Systems. El ON es un compuesto con una vida media muy corta, por lo que no es posible utilizar métodos convencionales de detección. En cambio, como la mayor parte del $\mathrm{ON}$ en el organismo se encuentra en forma de nitrito y nitrato, podemos utilizar la concentración de estos aniones para realizar una medida cuantitativa de la producción de ON. Para ello, primero se debe convertir el nitrato a nitrito $\mathrm{y}$, posteriormente, convertir este nitrito en un cromóforo, mediante la reacción de Griess, y realizar la medición espectrofotométrica a $550 \mathrm{~nm}$ $(20,21)$. La variabilidad de la técnica para determinación del $\mathrm{ON}$, tal y como aparece en el protocolo correspondiente es de 10 a 92 umoles por litro.

2) Determinación de la actividad oxidante: Mediante un protocolo que permite la determinación del malonildialdehído (MDA) como producto de la peroxidación lipídica, siguiendo la técnica del ácido tiobarbitúrico (TBA) y de las especies reactivas (TBARS). En primer lugar debe procederse a precipitar las proteínas y separar el MDA de las mismas. Después se hace reaccionar el MDA con el
TBA (1 hora en ebullición en oscuridad) y el resultado es la formación de un complejo que emite fluorescencia (fig. 1). Se extrae este complejo mediante butanol y a continuación se mide la fluorescencia del sobrenadante depositado en una placa multipocillos. La técnica está ya establecida y descrita extensamente en trabajos anteriores $(19,22,23)$.

3) Determinación del estado antioxidante total: Siguiendo una técnica enzimático-colorimétrica y mediante un preparado comercial de Laboratorios Randox S.L. En este procedimiento se induce la reacción de la ferrilmioglobina y el ABTS (2,2'azino-di-[3-etilbenzotiazolín sulfonato]) para obtener el radical catión ABTS, que presenta un color verde-azulado cuya intensidad puede medirse en espectrofotómetro a $600 \mathrm{~nm}$. La presencia de antioxidantes inhibe la formación de este catión, de modo que se producirá una reducción de la intensidad de dicha coloración, proporcional a la concentración de antioxidantes de la muestra (fig. 2). Esta técnica ha sido descrita con anterioridad, utilizada por nuestro grupo de investigación y validada en trabajos anteriores $(19,22,23)$.

Respecto al procesamiento de los datos, hay que enfatizar que el tamaño de la muestra se calculó mediante el programa Epiinfo StatCalc (Atlanta GA, USA). Todos los datos fueron registrados en una hoja diseñada para este particular en el progra-

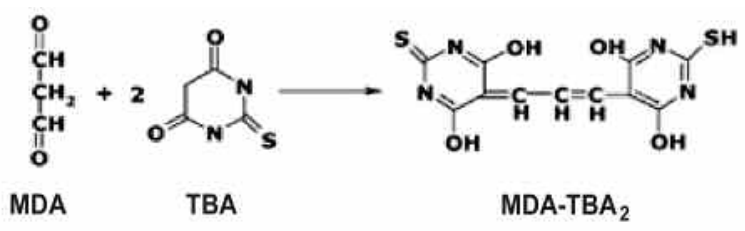

Fig. 1: Formación del complejo fluorescente a partir de malonildialdehído (MDA) y ácido tiobarbitúrico (TBA). 


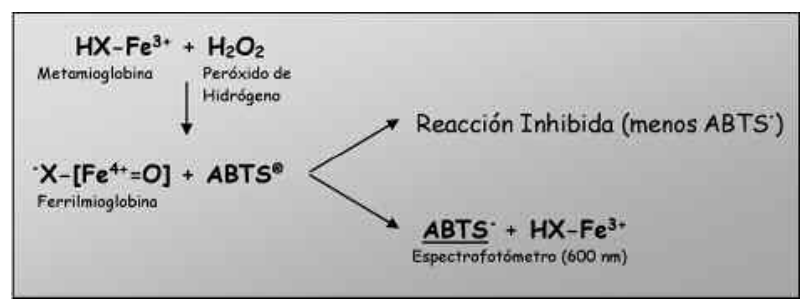

Fig. 2: Reacciones bioquímicas que tienen lugar durante la determinación del estado antioxidante total. ABTS=2,2'-Azino-di-[3-etilbenzotiazolin sulfonato]. $A O X=$ antioxidantes.

ma Excel (Microsoft Windows Professional, Microsoft Corporation, USA). El análisis de los resultados se llevó a cabo mediante el programa SPSS 15.0 (SPSS Inc, Chicago, IL, USA). Para la comparación de las variables cuantitativas se utilizó el estadístico t de Student (nivel de confianza 95\%), mientras que para el análisis de las variables cualitativas se empleó el chi cuadrado.

\section{RESULTADOS}

Las características de los sujetos del estudio se exponen en la tabla II. La distribución por sexos en ambos grupos fue homogénea, siendo la proporción entre hombres y mujeres de 0,765 en el GG y de 0,875 en el GC. El rango de edad estaba entre los 40 a los 90 años.

Las pruebas bioquímicas realizadas en los humores acuosos de los sujetos del estudio han aportado los siguientes datos:

1) Determinación del Óxido Nítrico Total: Los valores obtenidos fueron la media y desviación estándar (DE) de 2-3 medidas por paciente. Los resultados fueron significativamente superiores en el GG $(75,885 \mu \mathrm{M}$; DE 29,203 $\mu \mathrm{M})$ respecto del GC

Tabla II. Características demográficas de los grupos de estudio

$\begin{array}{ccc}\text { Grupo } & \text { Grupo } & \text { p-valor** } \\ \text { Glaucoma } & \text { Cataratas }\end{array}$

\begin{tabular}{lccc}
\hline $\mathrm{N}$ & 60 & 60 & - \\
Hombres & $26(43,3 \%)$ & $28(46,7 \%)$ & - \\
Mujeres & $34(56,7 \%)$ & $32(53,3 \%)$ & - \\
Índice de masculinidad* & 0,765 & 0,875 & \\
Edad & $76,4 \pm 6,7$ & $74,4 \pm 9,9$ & 0,207 \\
\hline \hline
\end{tabular}

* Índice Masculinidad: n. ${ }^{\circ}$ Hombres/n. ${ }^{\circ}$ Mujeres.

** Prueba T para muestras independientes.

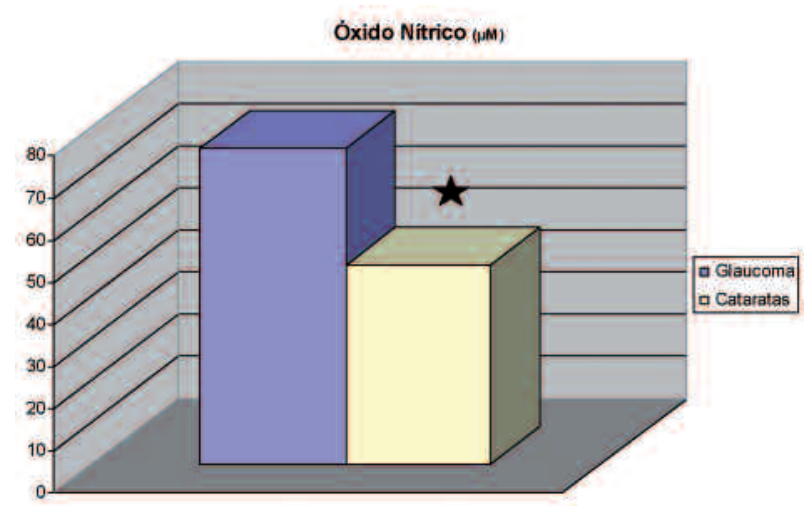

Fig. 3: Determinación de ON en el humor acuoso. La figura muestra los niveles de óxido nítrico $(N O)$ medidos en $\mu M$, que fueron significativamente superiores en el grupo de glaucoma $(p<0,05)$. El estadístico utilizado fue la mediana.

(58,020 $\mu \mathrm{M} ; 27,185 \mu \mathrm{M})$, siendo el p valor=0,0007. La mediana para ambos grupos viene expuesta en la figura 3.

2) Determinación de la actividad oxidativa (MDA/TBARS): El ensayo se repitió tres veces y los resultados son la media y DE de tres medidas por cada paciente. Se observaron unos valores estadísticamente significativos y superiores en el GG $(0,442 \mu \mathrm{M}$; DE $0,0159 \mu \mathrm{M})$ respecto del GC $(0,089$ $\mu \mathrm{M}$; DE 0,030 $\mu \mathrm{M})$, siendo el p valor=3,05551E-38. La figura 4 refleja la mediana para ambos grupos de experimentación.

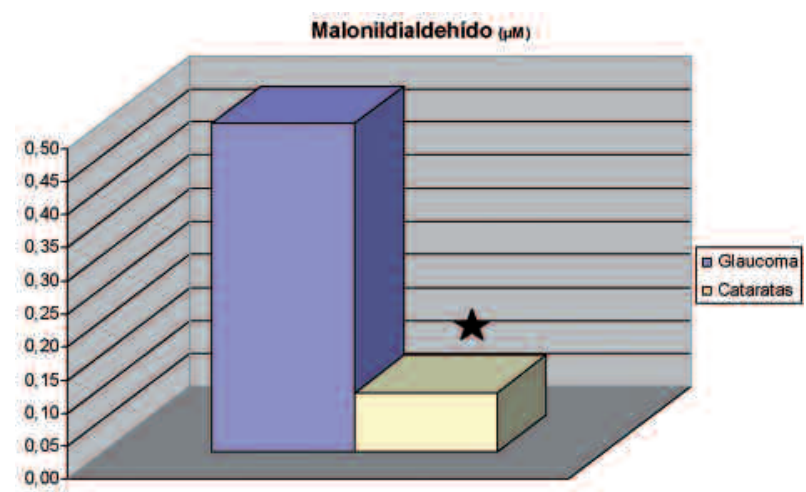

Fig. 4: Determinación de EROx mediante la peroxidación lipídica. Actividad oxidativa, medida como niveles de malonildialdehído (MDA, $\mu M)$ mediante la técnica del ácido tiobarbitúrico (TBARS). El estado oxidativo fue significativamente superior en el grupo de pacientes con glaucoma $(p<0,05)$. El estadístico utilizado fue la mediana. 
3) Determinación de Estado Antioxidante Total: Los resultados obtenidos son la media y DE de dos ensayos. El estado antioxidante total fue significativamente menor en el GG (1,954 mM; DE 0,623 $\mathrm{mM}$ ) respecto del GC (4,010 mM; DE 1,341 mM), siendo el valor de la $\mathrm{p}=2,97074 \mathrm{E}-19$. La mediana para ambos grupos se muestra en la figura 5 .

\section{DISCUSIÓN}

El presente estudio se ha diseñado para identificar moléculas que puedan intervenir en la patogenia y en la evolución del GPAA. Debido a la imposibilidad de reclutar un grupo de personas sanas para extraerles en el quirófano una muestra de humor acuoso, este estudio ha utilizado un grupo de pacientes con cataratas no complicadas como el grupo comparativo, Aunque la presencia de estrés oxidativo en las cataratas ha sido demostrado con anterioridad (17) y considerando que los valores de este grupo son el resultado del proceso de cataratogénesis, hemos partido de estos valores para comparar el nivel de estrés oxidativo obtenido de los pacientes glaucomatosos en estadío quirúrgico según los nomogramas de riesgo y el criterio del oftalmólogo, que fueron intervenidos mediante la técnica de trabeculectomía «ab externo» con los hallados en los sujetos sin glaucoma.

El estudio de la distribución por sexos y edad no mostró diferencias significativas entre los dos gru-

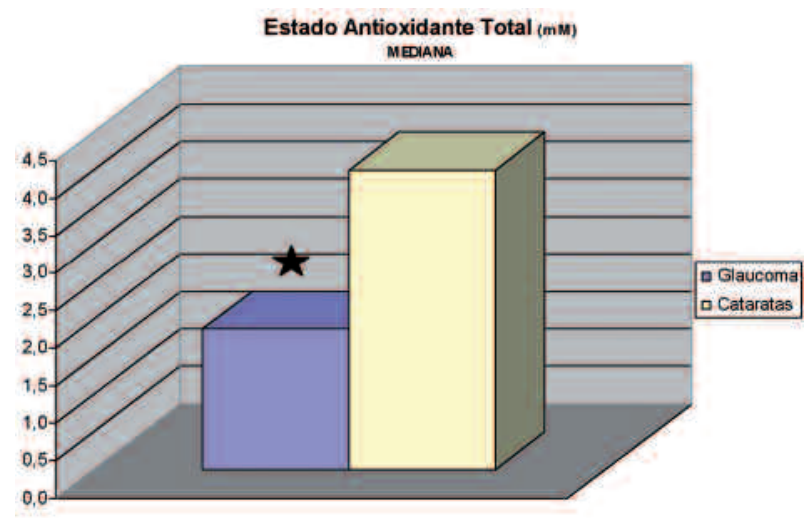

Fig. 5: Determinación de la actividad antioxidante. La figura muestra el estado antioxidante total, expresado en mM. En el grupo de glaucoma se observa una actividad global antioxidante significativamente menor que el observado en el grupo de cataratas $(p<0,05)$. El estadístico utilizado fue la mediana. pos. Aunque el rango de edades fue desde los 40 hasta los 90 años, la mayoría de los pacientes de este estudio se situaban por encima de los 60 años. Este dato resulta interesante en el contexto de nuestro trabajo, puesto que ya ha sido demostrado que tanto el glaucoma como las cataratas tienen una relación directa con el aumento de la edad y el envejecimiento (4). Un grupo de investigación del Instituto de Oftalmobiología Aplicada (IOBA) realizó un trabajo sobre una población de Segovia en la cual concluyen que la prevalencia del glaucoma en el grupo de personas entre 40-49 años era de 0,01\%, y en las personas entre 60-69 era de 2,40\% 25 .

Nuestros objetivos han sido determinar la presencia de ON, de EROx y el estado de los mecanismos antioxidantes (AOXT) en relación a la progresión del glaucoma, utilizando el humor acuoso obtenido al inicio de la cirugía programada de segmento anterior ocular. Para facilitar la lectura de esta sección vamos a estructurarla en los siguientes apartados:

1) Se han analizado los niveles de $\mathrm{ON}$ en el humor acuoso en ambos grupos de sujetos, hallando valores estadísticamente superiores en el grupo de pacientes con glaucoma frente al grupo de cataratosos. Estos resultados apoyan los obtenidos por Chang y colaboradores (13) que demuestran la variabilidad del $\mathrm{ON}$ en relación a la enfermedad glaucomatosa. No se han llevado a cabo experimentos para determinar la enzima implicada en la producción de $\mathrm{ON}$ en el humor acuoso de los sujetos del estudio, y por lo tanto no se puede establecer el fenotipo o fenotipos celulares que sobre-expresan el ON. Ahora bien, la concentración global de ON sí que está significativamente aumentada en los pacientes que han demostrado empeoramiento de la enfermedad glaucomatosa, siendo el criterio del oftalmólogo el que establece el riesgo de progresión y por tanto la conveniencia de la cirugía antiglaucomatosa. No se ha determinado la cantidad de $\mathrm{ON}$ que se transforma en peroxinitritos y por tanto no se puede asegurar la presencia del estrés nitrosativo. Aun así, considerando todos nuestros resultados proponemos que el aumento de la formación de ON en el humor acuoso en los pacientes con glaucoma, en presencia de oxidantes debe inducir la formación de peroxinitritos y como consecuencia es muy posible que se induzca el estrés nitrosativo. Este punto es un objetivo primordial a tratar en los experimentos futuros de esta línea de investigación.

2) Las determinaciones enzimático-colorimétricas de EROx y AOXT demuestran actividad red-ox 
en el humor acuoso de ambos grupos, siendo notable el mayor desequilibrio entre la formación de EROS y disminución de AOXT en el GG. Varios grupos de investigación han realizado estudios que demuestran la existencia de estrés oxidativo en el envejecimiento (24) y en relación al glaucoma, como el trabajo realizado por Ferreira y colaboradores (26) y, en este sentido, nuestros resultados señalan los mismos parámetros que los obtenidos por este grupo de investigadores, apoyando la presencia de agentes oxidantes en el humor acuoso en el glaucoma en estadio pre-quirúrgico. Este ha sido definido como la fase de la enfermedad en la que la progresión de las pruebas morfológicas (excavación papilar) y funcionales (campo visual, análisis de fibras ópticas) y/o la falta de respuesta al tratamiento hipotensor (o la falta de cumplimiento), independientemente de reflejar el fármaco utilizado, aconseja la cirugía antiglaucomatosa para evitar la pérdida de visión.

La neuropatía óptica glaucomatosa es una enfermedad neurodegenerativa y como tal es causa de ceguera irreversible de los pacientes afectos (4). Sabemos que, desde un punto de vista puramente mecánico su causa principal es el aumento de la presión en el interior del globo ocular. Pero, esta enfermedad se caracteriza por la inducción de apoptosis a las células ganglionares de la retina $(4,15)$. ¿Cuáles son, entonces, las causas celulares y moleculares que rodean la degeneración de las células ganglionares? ¿Es este un hecho restringido a la retina? ¿Existe la posibilidad de frenar o invertir el proceso degenerativo que conduce a la ceguera? Como es obvio, son aún demasiadas las preguntas sin respuesta. En nuestro estudio, reforzamos los hallazgos de Ferreira y cols. (26), y avalamos la implicación del ON y del estrés oxidativo como mecanismos etiopatogénicos del glaucoma, enfatizando la importancia de los marcadores bioquímicos en humor acuoso para evaluar la progresión de la neuropatía óptica glaucomatosa.

De todo ello, se deduce que el conocimiento de las bases bioquímicas y moleculares que constituyen la etiopatogenia del glaucoma en humanos es esencial para investigar nuevas vías de tratamiento que puedan mejorar las actuales. Además, la comprensión de los mecanismos implicados en las funciones celulares, incluyendo la muerte y la supervivencia neuronal y, si es posible, la identificación de cualquier proceso que pueda informar acerca de reparación celular y reposición de elementos daña- dos durante la enfermedad glaucomatosa, abriría nuevos campos en la expectativa de visión de los pacientes con glaucoma, que sería los beneficiarios inmediatos de estas investigaciones, incluyendo las estrategias terapéuticas que permitan controlar la disponibilidad de ON y la inhibición en la formación o el bloqueo de la actividad de las EROx, favoreciendo el estado de las defensas antioxidantes.

\section{BIBLIOGRAFÍA}

1. Macknight AD, McLaughlin CW, Peart D, Purves RD, Carre DA, Civan MM. Formation of the aqueous humor. Clin Exp Pharmacol Physiol 2000; 27: 100-106.

2. Quigley HA. Number of people with glaucoma worldwide. Br J Ophthalmol 1996; 80: 389-393.

3. Fuchsjager-Mayrl G, Wally B, Georgopoulos M, Rainer $G$, Kircher K, Buehl W, et al. Ocular blood flow and systemic blood pressure in patients with primary open-angle glaucoma and ocular hypertension. Invest Ophthalmol Vis Sci 2004; 45: 834-839.

4. Nicoara S. Modern concepts in the pathogenesis and treatment of glaucoma. Oftalmologia 2000; 51: 9-13.

5. Lancaster JR Jr, Hibbs JB Jr. EPR demonstration of ironnitrosyl complex formation by cytotoxic activated macrophages Proc Natl Acad Sci U S A. 1990; 87: 1223-1227.

6. Khatsenko OG, Gross SS, Rifkind AB, Vane JR. Nitric oxide is a mediator of the decrease in cytochrome P450dependent metabolism caused by immunostimulants. Proc Natl Acad Sci USA 1993; 90: 11147-11151.

7. Palmer RM, Ferrige AG, Moncada S. Nitric oxide release accounts for the biological activity of endothelium-derived relaxing factor. Nature 1987; 327: 524-526.

8. Furchgott RF, Zawadzki JV. The obligatory role of the endothelial cells in the relaxation of arterial smooth muscle by acetylcholine. Nature 1980; 288: 373-376.

9. Rapoport RM, Draznin MB, Murad F. Endothelium dependent relaxation in rat aorta may be mediated through cyclic GMP-dependent protein phosphorylation. Nature 1983; 306: 174-176.

10. Ignarro LJ, Buga GM, Wood KS, Byrns RE, Chaudhuri G. Endothelium-derived relaxing factor produced and released from artery and vein is nitric oxide. Proc Natl Acad Sci U S A 1987; 84: 9265-9269.

11. Gerkowicz M, Kosior-Jarecka E, Koziol-Montewka M. Role of nitric oxide in ophthalmic diseases. Klin Oczna 2005; 107: 533-536.

12. Kim JC, Park GS, Kim JK, Kim YM. The role of nitric oxide in ocular surface cells. J Korean Med Sci 2002; 17: 389-394.

13. Chang CJ, Chiang CH, Chow JC, Lu DW. Aqueous humor nitric oxide levels differ in patients with different types of glaucoma. J Ocul Pharmacol Ther 2000; 16: 399-406.

14. Fiers W, Beyaert R, Declercq W, Vandenabeele P. More than one way to die: apoptosis, necrosis and reactive oxygen damage. Oncogene 1999; 18: 7719-7730.

15. Nicholls DG, Budd SL. Mitochondria and neuronal survival. Physiol Rev 2000; 80: 315-360. 
16. Hayes JD, McLellan LI. Glutathione and glutathionedependent enzymes represent a co-ordinately regulated defense against oxidative stress. Free Radic Res 1999; 31 : 273-300.

17. Ottonello S, Foroni C, Carta A, Petrucco S, Maraini G. Oxidative stress and age-related cataract. Ophthalmologica 2000; 214: 78-85.

18. Ethen CM, Reilly $C$, Feng X, Olsen TW, Ferrington DA. Age-related macular degeneration and retinal protein modification by 4-hydroxy-2-nonenal. Invest Ophthalmol Vis Sci 2007; 48: 3469-3479.

19. Verdejo C, Marco P, Renau-Piqueras J, Pinazo-Duran MD. Lipid peroxidation in proliferative vitreoretinopathies. Eye 1999; 13: 183-188.

20. Kawano K, Masuda H, Yano M, Kihara K, Sugimoto A, Azuma H. Altered nitric oxide synthase, arginase and ornithine decarboxylase activities, and polyamine synthesis in response to ischemia of the rabbit detrusor. J Urol 2006; 176: 387-393.

21. Giustarini D, Rossi R, Milzani A, Dalle-Donne I. Nitrite and nitrate measurement by griess reagent in human plas- ma: evaluation of interferences and standardization. Methods Enzymol 2008; 440: 361-380.

22. Pyles LA, Fortney JE, Kudlak JJ, Gustafson RA, Einzig S. Plasma antioxidant depletion after cardipulmonary bypass in operations for congenital heart disease. $J$ Thorac Cardiovasc Surg 1995; 110: 165-171.

23. Yasar M, Yildiz, S, Mas R, Dundar K, Yildirim A, Korkmaz $A$, et al. The effect of hyperbaric oxygen treatment on oxidative stress in experimental acute necrotizing pancreatitis. Physiol Res 2003; 52: 111-116.

24. Aguirre F, Martin I, Grinspon D, Ruiz, M, Hager A, De Paoli T, et al. Farach HA, Poole Jr CP. Oxidative damage, plasma antioxidant capacity, and glucemic control in elderly NIDDM patients. Free Radic Biol Med 1998; 24: 580-585.

25. Anton A, Andrada MT, Mujica V, Calle MA, Portela J, Mayo A. Prevalence of primary open-angle glaucoma in a Spanish population: the Segovia study. J Glaucoma 2004; 13: 371-376.

26. Ferreira SM, Lerner SF, Brunzini R, Evelson PA, Llesuy $S$. Oxidative stress markers in aqueous humour of glaucoma patients. Am J Ophthalmol 2004; 137: 62-69. 